\title{
Sinoaortic baroreceptor reflex arc modulation of nociception in spontaneously hypertensive and normotensive rats
}

\author{
ALAN RANDICH \\ University of Iowa, Iowa City, Iowa
}

\begin{abstract}
Spontaneously hypertensive rats of the Okamoto-Aoki strain (SHR) and normotensive rats of the Wistar-Kyoto strain (WKY) were assessed in hot-plate and flinch-jump analgesimetric assays. SHRs manifested longer latencies than WKYs to both paw-lick and jump in response to painful thermal stimulation in the hot-plate assay, but SHRs had lower flinch and jump thresholds than WKYs to electric shock stimulation in the flinch-jump assay. Bilateral sinoaortic deafferentation did not alter response latencies of either SHRs or WKYs in the hotplate assay, but significantly lowered flinch and jump thresholds of both SHRs and WKYs in the flinch-jump assay. These findings are discussed in terms of baroreceptor reflex arc modulation of endogenous pain-regulatory systems and motor control.
\end{abstract}

Animal models of hypertension have not only provided information regarding the etiology of hypertension in humans, but also suggested that blood pressure control systems interact with pain-regulatory systems. Antinociceptive behaviors are manifested by rats with experimentally induced renal hypertension, desoxycorticosterone acetate (DOCA) salt-induced hypertension, and genetic hypertension in hot-plate and paw-pinch analgesimetric assays (Maixner, Touw, Brody, Gebhart, \& Long, 1982; Randich, 1981; Randich \& Maixner, 1981; Zamir \& Segai, 1979; Zamir, Simantov, \& Segal, 1980). In each of these forms of hypertension, the antinociception is attenuated following peripheral administration of the opiate receptor antagonist naloxone (Maixner et al., 1982; Zamir et al., 1980), thereby implicating the involvement of endogenous opioid pain-inhibition systems. This role of endogenous opioids is also supported by findings that (1) experimental renal and Sabra hypertensive rats (SBH) have elevated levels of opioids in the cervical region of the spinal cord (Zamir et al., 1980), (2) spontaneously hypertensive rats of the Okamoto-Aoki (SHR) strain have increased levels of whole brain opioid receptors (Martucci \& Hahn, 1980), and (3) SBH rats show reduced binding of $\left({ }^{3} \mathrm{H}\right)$ naloxone in the hippocampus and dorsal horn of the spinal cord (Zamir, Segal, \& Simantov, 1981). In accord with the above observations, young and adult humans with essential hypertension show higher sensory and pain thresholds than age-matched normotensive humans in response to electrical stimulation of tooth pulp (Zamir \& Shuber, 1980).

This research was supported by an NIMH grant (MH 15773) to A. Randich. Address reprint requests to A. Randich, Department of Psychology, University of Iowa, Iowa City, Iowa 52242.
The cardiopulmonary baroreceptor reflex arc appears to be critical for the antinociception of SHRs in the hot-plate assay, since either administration of hexamethonium or resection of the right vagus (which carries cardiopulmonary afferents) reduces the antinociception (Maixner et al., 1982). In SHRs, cardiopulmonary baroreceptors are "reset" or have increased threshold and reduced sensitivity, as measured by the slope of the linear portion of the steady state impulse frequency-pressure curve (Brown, 1980; Thorén, 1979). However, decreased venous and left atrial distensibility are believed to compensate for this resetting, resulting in greater tonic and possibly stress-induced activity of the cardiopulmonary baroreceptors in SHRs compared with that in normotensive WKY controls (Ricksten, Noresson, \& Thorén, 1979; Thorén, 1979; Thorén, Noresson, \& Ricksten, 1979). Thus, it is possible that peripheral increases in blood pressure that activate cardiopulmonary baroreceptors may induce antinociception to painful thermal stimuli, and that this mechanism is augmented in the SHR. Electrophysiological data showing that stimulation of vagal C-fibers in normotensive rats inhibits neural activity evoked by noxious stimulation at several levels of the neuroaxis, including cunneate nucleus and spinoreticular tracts, are compatible with this view (Dell, 1972; Gahery \& Vigier, 1974; Thies \& Foreman, 1981). However, these findings do not preclude the possibility that activation cardiopulmonary baroreceptors produces such behaviors through influences on motor excitability (Deshpande \& Devanandan, 1970; Ginzel, 1973a, 1973b).

In contrast, the sinoaortic baroreceptor reflex arc does not appear critical for the antinociception manifested by SHRs in the hot-plate assay, since bilateral sinoaortic deafferentation has no effect on the antinociception (Maixner et al., 1982). However, the 
sinoaortic baroreceptor reflex arc may modulate nociception to forms of noxious stimulation other than painful thermal stimulation. Acute phenylephrineinduced hypertension attenuates wheel-turn escape/ avoidance responding evoked by noxious trigeminal stimulation, and this effect is reversed following bilateral sinoaortic deafferentation (Dworkin, Filewich, Miller, Craigmyle, \& Pickering, 1979). Carotid sinus distension is also known to activate cells in the nucleus reticularis gigantocellularis and nucleus reticularis parvocellularis-two areas associated with transmission of information about pain (Biscoe \& Sampson, 1970a, 1970b; Hammond, 1980). Although there are no data on sinoaortic baroreceptor reflex arc modulation of nonthermal noxious stimuli in the SHR, the fact that sinoaortic baroreceptors are reset in the SHR (Thorén, 1979) suggests that the SHR may be hyperalgetic to any such stimuli compared with normotensive WKY controls, since there is no compensation for such resetting.

Therefore, the present experiments were carried out to both replicate and extend the nociceptive profile of SHRs and WKYs, and to further characterize possible interactions between blood pressure control and pain-regulatory systems. This was accomplished by assessing (1) basal levels of responsivity of SHRs and WKYs to both painful thermal stimulation and electric footshock stimulation in the hot-plate and flinch-jump assays, and (2) the effect of bilateral sinoaortic deafferentation in the control of such behaviors.

\section{METHOD}

\section{Subjects}

Twenty-one experimentally naive male spontaneously hypertensive rats (SHRs) of the Okamoto-Aoki strain and 23 experimentally naive male Wistar-Kyoto normotensive rats (WKYs) served as subjects. The rats were $180-185$ days of age at the start of the experiment. The rats were obtained from an inbred brother-sister colony maintained by the Cardiovascular Research Group at the University of Iowa. Each rat was housed in an individual wire-mesh cage and maintained on ad-lib food and water. Light onset in the colony room was at $0700 \mathrm{~h}$ and light termination was at $1900 \mathrm{~h}$.

\section{Apparatus}

The hot-plate apparatus consisted of a circular copper plate $(20 \mathrm{~cm}$ in diameter) immersed in a water bath. The temperature of the plate was maintained at $55^{\circ} \pm .05^{\circ} \mathrm{C}$ by a constant temperature control unit. The copper plate was enclosed by a $45-\mathrm{cm}$ Plexiglas cylinder which prevented escape from the hot-plate.

The flinch-jump apparatus consisted of a $26 \times 23 \times 30 \mathrm{~cm}$ chamber constructed of wood. Three side walls and the top were painted gray. The front of the chamber was clear Plexiglas. Shock was delivered to the grid floor of the chamber by a highvoltage, high-resistance shock source. All shock presentations were controlled by solid state timing devices.

\section{Procedure}

SHRs and WKYs were initially tested for basal responsivity to the thermal stimulus in the hot-plate assay. Each rat was re- moved from a holding cage and placed on the copper hot-plate. The latency to either lick a hindpaw or jump with all four paws off the plate was recorded.

On the following day, the same rats were tested for basal responsivity to the electric shock stimulus in the flinch-jump assay using a modified version of Evans (1961). Each rat was placed in the chamber and presented with a single ascending series of electric shocks. The initial shock intensity was 0.00 -mA and incremented in .05-mA steps. The intershock interval was approximately $30 \mathrm{sec}$, but depended upon the rat's maintaining all four paws in contact with the grid floor. All shocks were $100 \mathrm{msec}$ in duration. A flinch response was defined as the lowest shock intensity that elicited a withdrawal of a single forepaw from the shock grids. A jump response was defined as the lower of two successive shock intensities that elicited simultaneous withdrawal of both hindpaws from the shock grids. Since only a single ascending shock sequence was presented, the present procedure tends to overestimate true response thresholds. However, this procedure was adopted in order to minimize total exposure to shock, since, in our laboratory, repeated exposure to ascending and descending shock sequences results in conditioning of apparatus cues, which interferes with accurate assessments of thresholds.

Following these initial tests of nociception, all rats received either a bilateral sinoaortic deafferentation (see protocols below) or a sham operation. The rats were permitted a 3-week recovery period and were then retested in the manner described above, that is, hot-plate assay followed by flinch-jump assay.

\section{Surgical Procedures}

The bilateral sinoaortic deafferentations were carried out according to the method of Krieger (1964). A 3-cm midline incision was made in the neck, exposing the underlying muscles. The vagus and the carotid artery were separated from the sympathetic trunks and neurovascular sheath. The superior laryngeal nerve was resected. The sympathetic trunk was also resected just inferior to the superior cervical ganglion. Denervation of the carotid sinus baroreceptors was accomplished by stripping the carotid bifurcation of all fibers and extraneous tissue, and then painting the bifurcation with $10 \%$ phenol in ethanol. The operation was carried out bilaterally in a single-stage operation under ether anesthesia.

Sham-operated rats received the identical operation except for resections of the superior laryngeal, sympathetic trunk, and stripping and painting of the carotid bifurcation. As noted previously, all rats received a 3-week postoperative recovery period.

The test for complete sinoaortic baroreceptor deafferentation was conducted following all experimental testing. The test involved a bolus injection of phenylephrine via an indwelling catheter (PE 50) implanted in the femoral vein. The absence of reflex bradycardia in response to a $50-\mathrm{mm}-\mathrm{Hg}$ increase in arterial blood pressure was considered as evidence for a complete sinoaortic deafferentation. Any rat showing reflex bradycardia to this test was eliminated from analysis. This test was conducted $24 \mathrm{~h}$ after implantation of arterial and venous femoral catheters while the rat was conscious and moving freely in a holding cage.

On the basis of the phenylephrine tests and mortality rates, data from the following number of rats were included from the hot-plate and flinch-jump retests: WKY shams $=10$; WKY SADs = 6; SHR shams = 8; and SHR SADs = 6. (SAD refers to bilateral sinoaortic deafferentation.)

\section{RESULTS}

The results of the initial tests of responsivity to the thermal stimulus in the hot-plate assay and the electric shock stimulus in the flinch-jump assay are presented in Figures 1 and 2, respectively. 


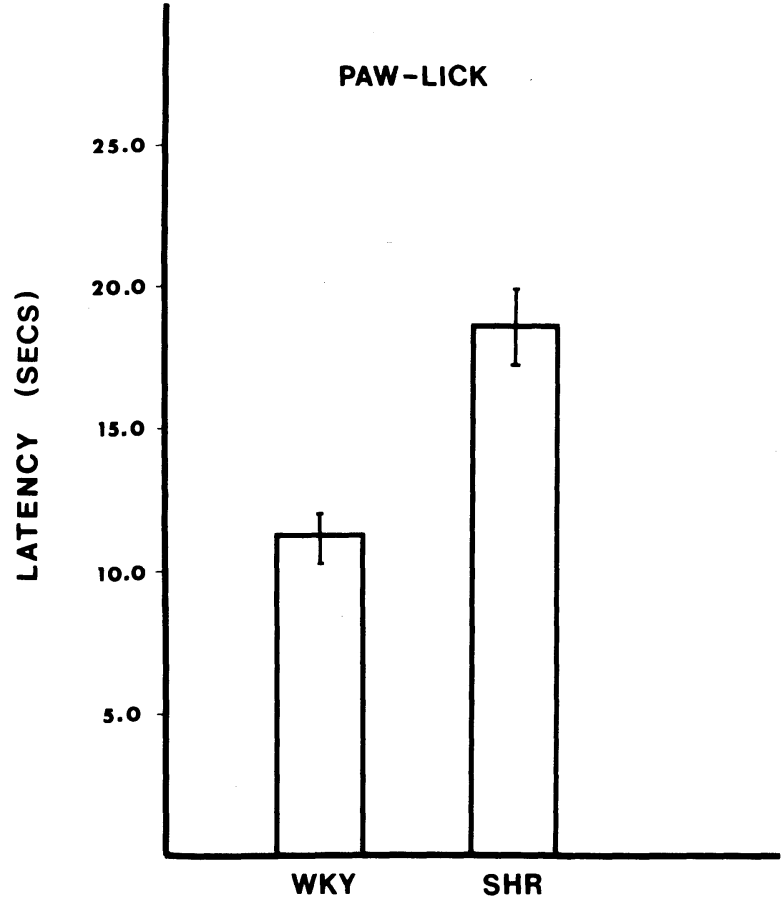

Figure 1. Mean latencies ( \pm SEM) of SHRs and WKYs to either paw-lick or jump in the hot-plate assay $\left(55^{\circ} \pm .05^{\circ} \mathrm{C}\right)$.

Figure 1 shows that SHRs had longer latencies than WKYs to either paw-lick or jump in response to the thermal stimulation. These impressions were confirmed by an analysis of variance that indicated a significant between-groups difference in response latencies $[F(1,42)=28.81]$. Therefore, these outcomes replicate previous findings (Maixner et al., 1982; Zamir \& Segal, 1979).

In contrast to the antinociception manifested by SHRs in the hot-plate assay, Figure 2 reveals that
SHRs were more responsive than WKYs to electricshock stimulation in the flinch-jump assay. Specifically, SHRs had significantly lower flinch thresholds than WKYs $[F(1,42)=6.23]$ and significantly lower jump thresholds than WKYs $[F(1,42)=8.78]$. In summary, SHRs can be viewed as hypoalgetic with respect to painful thermal stimulation, but hyperalgetic with respect to electric-shock stimulation.

Figures 3, 4, and 5 present the effects of either bilateral SADs or sham operations on nociception in the hot-plate, flinch, and jump retests, respectively. The pretest measures reported in each figure were obtained from the initial tests reported above.

Figure 3 indicates that bilateral SAD had no major effect upon latencies to respond to the thermal stimulus in either SHRs or WKYs. These data were analyzed with an analysis of variance with between factors of strain (SHR vs. WKY) and operation (sham vs. SAD), and a within factor of trials (preoperation versus postoperation). This analysis revealed only a significant effect of strain $[F(1,23)=$ 43.21], indicating that SHRs manifested longer response latencies than WKYs.

Figure 4 reveals that the bilateral SAD lowered flinch thresholds in both SHRs and WKYs, but that the sham operation had no major effect. An analysis of variance identical to that described above indicated a significant effect of strain $[F(1,26)=14.69]$, a significant effect of the operation $[F(1,26)=10.31]$, and a significant trials effect $[F(1,26)=6.98]$. Thus, SHRs had lower flinch thresholds than WKYs, and the effect of the bilateral SAD was to lower flinch thresholds in both SHRs and WKYs relative to their sham-operated controls.

Figure 5 reveals that the bilateral SAD also lowered jump thresholds in both SHRs and WKYs. An analysis of variance indicated a significant effect of strain $[F(1,26)=13.95]$, a significant effect of the

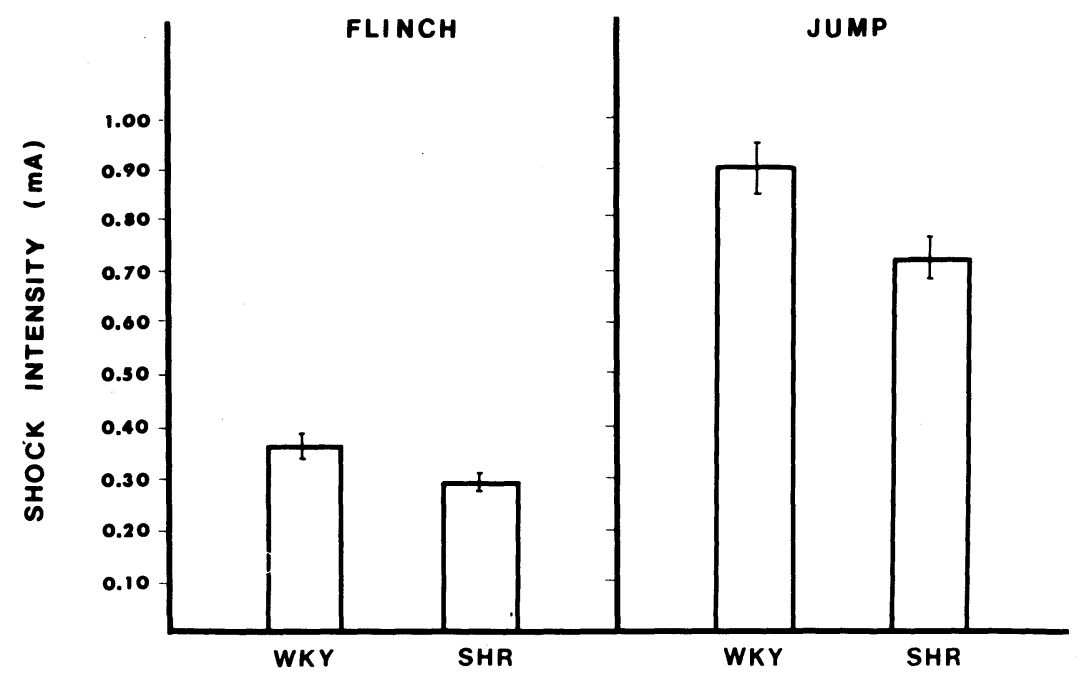

Figure 2. Mean flinch (left panel) and jump (right panel) thresholds of SHRs and WKYs in the flinch-jump assay. SEMs are denoted by vertical bars. 


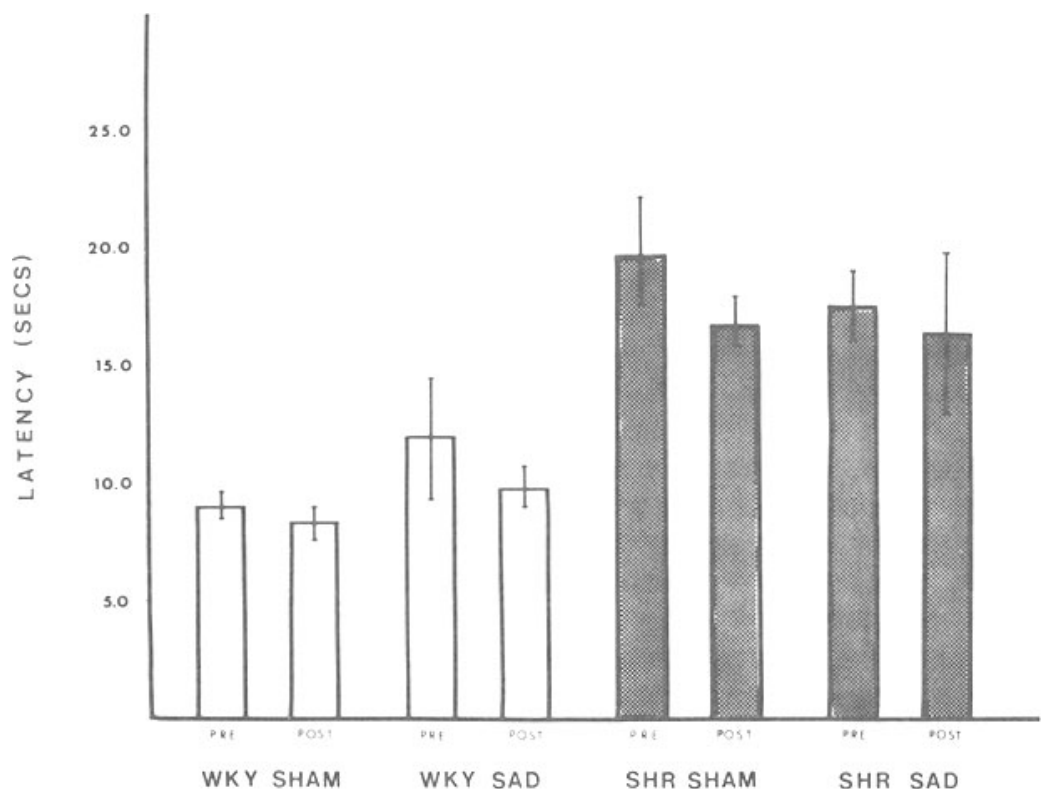

Figure 3. Mean latencies ( \pm SEM) of SHRs and WKYs to either paw-lick or jump in the hot-plate assay following either sham-operations or bilateral SADs.

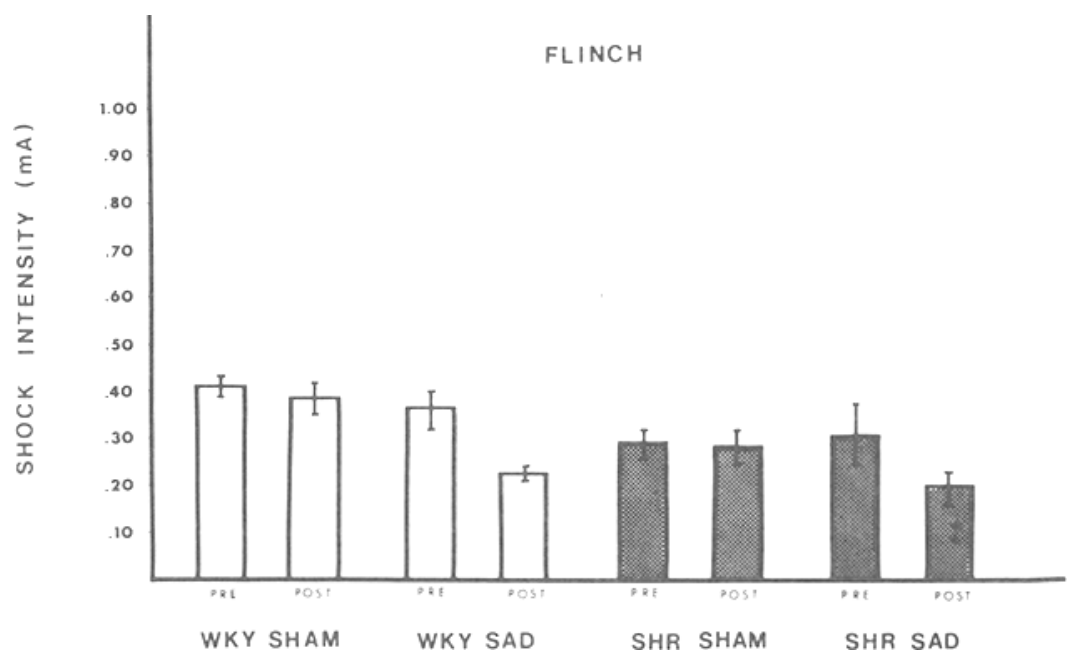

Figure 4. Mean flinch thresholds ( $($ SEM) of SHRs and WKYs following either shamoperations or bilateral SADs.

operation $[F(1,26)=4.77]$, and a significant effect of trials $[F(1,26)=5.72]$. Thus, SHRs had lower jump thresholds than WKYs, and the bilateral SAD lowered jump thresholds in both SHRs and WKYs relative to their sham-operated controls.

\section{DISCUSSION}

In accord with previous findings, SHRs manifested greater antinociception than WKYs did in response to painful thermal stimulation in the hotplate assay (Maixner et al., 1982; Randich, 1981;
Randich \& Maixner, 1981; Zamir \& Segal, 1979). This antinociception was unaffected by bilateral SAD, thereby confirming the report of Maixner et al. (1981). Thus, these findings are consistent with the view that antinociception of the SHR in response to thermal stimulation is modulated by the cardiopulmonary baroreceptor reflex arc, since resection of the right vagus is effective in reducing the antinociception (Maixner et al., 1982).

In contrast, the sinoaortic baroreceptor reflex arc may modulate nociception to electric shock stimulation. SHRs showed lower flinch and jump thresholds 


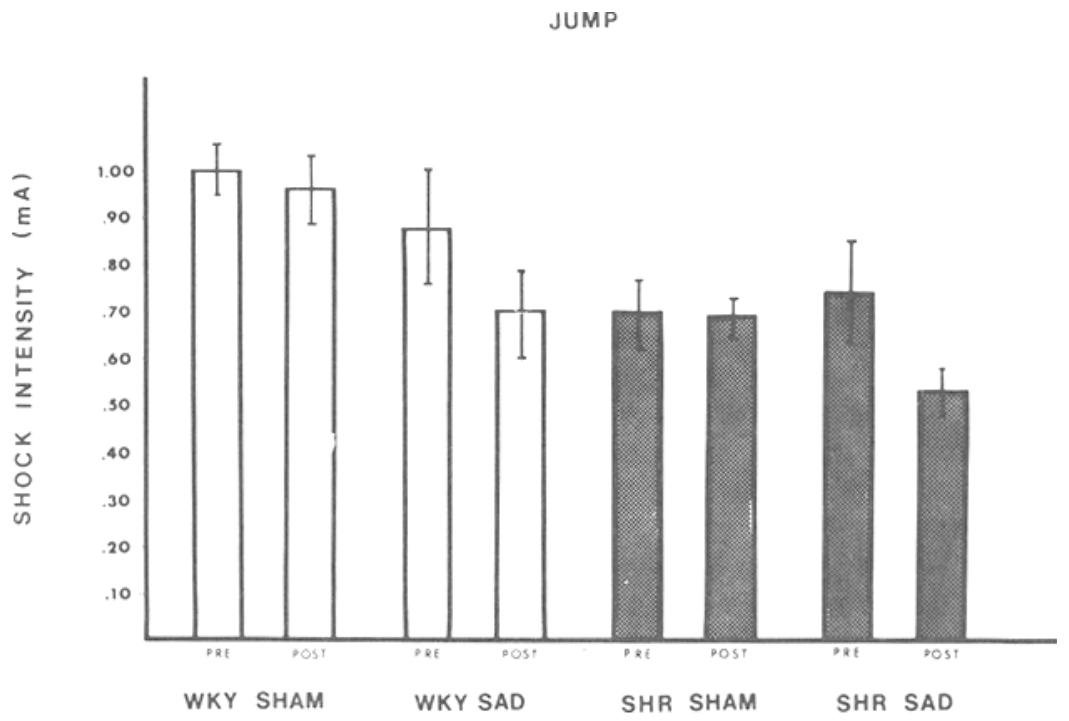

Figure 5. Mean jump thresholds ( \pm SEM) of SHRs and WKYs following either sham operations or bilateral SADs.

than age-matched normotensive WKYs during exposure to a single ascending sequence of shock intensities. It is important to note, however, that a flinch response was defined in terms of withdrawal of a single forepaw from the grids, and a jump response was defined in terms of simultaneous withdrawal of both hindpaws from the grid. It cannot be determined at the present time whether such differences between SHRs and WKYs would be maintained using other criteria, for example, defining flinch responses as any abrupt gross body movements or including "prancing" (rapid alternation of hindpaws) as a jump response. Bilateral SAD significantly lowered flinch and jump thresholds in both SHRs and WKYs compared with sham-operated controls. These outcomes are consistent with the view that sinoaortic baroreceptor resetting in the SHR may diminish the capacity of this reflex arc to inhibit pain induced by exposure to electric shock, and that the effectiveness of the bilateral SAD in reducing flinch and jump thresholds in both SHRs and WKYs stems from completely eliminating sinoaortic baroreceptor modulation of pain-regulatory systems. A similar account of sinoaortic baroreceptor reflex arc modulation of aversive trigeminal stimulation has been provided by Dworkin et al. (1979).

This view predicts that the degree of hyperalgesia observed in the SHR in response to shock would be directly related to the degree of sinoaortic baroreceptor resetting. As such, this behavior may serve as a valuable marker variable for either a propensity to become hypertensive or an existing state of hypertension in SHRs and may show age-dependency. It might also account for several recent reports that
SHRs acquire conditioned avoidance (Campbell \& DiCara, 1977; Sutterer, Perry, \& DeVito, 1980) and conditioned suppression of responding (Randich \& Maixner, 1981) at a faster rate than normotensive WKY controls. Specifically, these acquisition differences may not reflect learning differences between strains, but, rather, differences in the aversiveness of the electric shock unconditioned stimulus.

These studies do not, however, preclude an interpretation based upon baroreceptor reflex arc modulation of motor systems. Activation of either cardiopulmonary (Deshpande \& Devanandan, 1970; Ginzel, 1973a, 1973b) or carotid sinus (Coleridge, Coleridge, \& Rosenthal, 1976) baroreceptors produces a decrease in motor excitability, for example, inhibition of both flexor and extensor reflexes in cats. Thus, if resetting of sinoaortic baroreceptors in the SHR resulted in an augmentation of motor excitability, then the outcomes of the flinch-jump assay would be consistent with an interpretation based upon motor excitability.

If the sinoaortic and cardiopulmonary baroreceptor arcs are critical for modulating nociception in the rat, then the dissociation observed in terms of effects upon responsivity to various forms of noxious stimulation remains to be explained. Little evidence is available regarding differential specificity of cardiopulmonary and sinoaortic connections in the CNS, although physiological studies of carotid sinus, aortic depressor, and vagal influences on neuronal activity (Calaresu \& Ciriello, 1980; Gahery \& Vigier, 1974) and depressor responses (Ward \& Gunn, 1976) support such a notion. Clearly, a delineation of baroreceptor influences on nociception 
requires further anatomical specification of CNS pathways (cf. Galosy, Clark, Vasko, \& Crawford, 1981).

Finally, these outcomes support the general view that blood pressure control systems are physiologically linked to endogenous pain regulatory systems. The network formed by these systems may govern the elaboration of adaptive responses to physical and psychological stressors, and hence represent a dynamic physiological substrate vital to the existence of an organism.

\section{REFERENCES}

Biscoe, T. J., \& SAmpson, S. R. Field potentials evoked in the brain stem of the cat by stimulation of the carotid sinus, glossopharyngeal, aortic, and superior laryngeal nerves. Journal of Physiology (London), 1970, 209, 241-258. (a)

Biscoe, T. J., \& Sampson, S. R. Responses of cells in the brain stem of the cat to stimulation of the sinus, glossopharyngeal, aortic and superior laryngeal nerves. Journal of Physiology (London), 1970, 209, 359-373. (b)

Brown, A. M. Receptors under pressure: An update on baroreceptors. Circulation Research, 1980, 46, 1-10.

Calaresu, F. R., \& Ciriello, J. Projections to the hypothalamus from buffer nerves and nucleus tractus solitarius in the cat. American Journal of Physiology, 1980, 239, R130-R136.

Campbell, R. J., \& DiCARA, L. V. Running-wheel avoidance behavior in the Wistar/Kyoto spontaneously hypertensive rat. Physiology \& Behavior, 1977, 19, 473-480.

Coleridge, H. M., Coleridge, J. C., \& Rosenthal, F. Prolonged inactivation of cortical pyramidal tract neurons in cats by distension of the carotid sinus. Journal of Physiology (London), 1976, 256, 635-649.

DELl, P. Nucleus fasciculus solitarius activity: Visceral afferents and somatic functions. In C. Hockman \& C. C. Thomas (Eds.), Limbic system mechanisms and autonomic function. Springfield, Ill: Thomas, 1972.

Deshpande, S. S., \& Devanandan, M. S. Reflex inhibition of monosynaptic reflexes by stimulation of Type $\mathrm{J}$ pulmonary endings. Journal of Physiology (London), 1970, 206, 345-357.

Dworkin, B. R., Filewich, R. J., Miller, N. E., Craigmyle, N., \& Pickering, T. G. Baroreceptor activation reduces reactivity to noxious stimulation: Implications for hypertension. Science, 1979, 205, 1299-1301.

Evans, W. O. A new technique for the investigation of some analgesia drugs on the reflexive behavior in the rat. Psychopharmacologia, 1961, 2, 318-325.

GAHERY, Y., \& VigieR, D. Inhibitory effects in the cunneate nucleus produced by vagoaortic afferent fibers. Brain Research, 1974, 75, 241-246.

Galosy, R. A., Clarke, L. K., Vasko, M. R., \& Crawford, I. L. Neurophysiology and neuropharmacology of cardiovascular regulation and stress. Neuroscience \& Biobehavioral Reviews, 1981, 5, 137-175.

GinzeL, K. H. Muscle relaxation by drugs which stimulate sensory nerve endings $-\mathrm{I}$. The effects of veratrum alkaloids, phenyldiguanide and 5-hydroxytryptamine. Neuropharmacology, 1973, 12, 133-148. (a)

GINZEL, K. H. Muscle relaxation by drugs which stimulate sensory nerve endings-II. The effect of nicotinic agents. Neuropharmacology, 1973, 12, 149-164. (b)

HAmmond, G. R. Lesions of the medial medullary reticular area in rats impair CER acquisition. Physiological Psychology, $1980,8,483-486$.

Krieger, E. M. Neurogenic hypertension in the rat. Circulation Research, 1964, 15, 511-521.

Maixner, W., Touw, K. B., Brody, M. J., Gebhart, G. F., \& Long, J. P. Factors influencing the altered pain perceptions in the spontaneously hypertensive rat. Brain Research, 1982, 237, 137-145.

Martucci, C. P., \& Hahn, E. F. Brain opiate receptor concentrations are increased in adult spontaneously hypertensive rats. Endocrinology Research Communications, 1980, 6, 291-297.

RANDich, A. Nociceptive responses of spontaneously hypertensive and Wistar-Kyoto normotensive rats. Society for Neurosciences, 1981, 7, 735. (Abstract)

RANDich, A., \& MaIXNER, W. Acquisition of condition suppression and responsivity to thermal stimulation in spontaneously hypertensive, renal hypertensive, and normotensive rats. Physiology \& Behavior, 1981, 27, 585-590.

Ricksten, S. E., Noresson, E., \& Thorén, P. Inhibition of renal sympathetic nerve traffic from cardiac receptors in normotensive and spontaneously hypertensive rats. Acta Physiologica Scandinavica, 1979, 106, 17-22.

Sutterer, J. R., Perry, J., \& DeVito, W. Two-way shuttle box and leverpress avoidance in spontaneously hypertensive and normotensive rat. Journal of Comparative and Physiological Psychology, 1980, 94, 155-163.

Thies, R., \& Foreman, R. D. Descending inhibition of spinal neurons in the cardiopulmonary region by electrical stimulation of vagal afferent nerves. Brain Research, 1981, 207, 178-183.

Thoren, P. Role of cardiac vagal C-fibers in cardiovascular control. Reviews of Physiology, Biochemistry, and Pharmacology, 1979, 86, 1-94.

Thoren, P., Noresson, E., \& Ricksten, S. E. Resetting of cardiac C-fibres endings in the spontaneously hypertensive rat. Acta Physiologica Scandinavica, 1979, 107, 13-18.

WARD, D. G., \& GunN, C. G. Locus coeruleus complex: Differential modulation of depressor mechanisms. Brain Research, $1976,107,407-411$.

ZAmir, N., \& SEGAL, M. Hypertension-induced analgesia: Changes in pain sensitivity in experimental hypertensive rats. Brain Research, 1979, 160, 170-173.

Zamir, N., Segal, M., \& Simantov, R. Opiate receptor binding in the brain of the hypertensive rat. Brain Research, 1981, 213, 217-222.

ZAMIR, N., \& Shuber, E. Altered pain perception in hypertensive humans. Brain Research, 1980, 201, 471-474.

Zamir, N., Simantov, R., \& Segal, M. Pain sensitivity and opioid activity in genetically and experimentally hypertensive rats. Brain Research, 1980, 184, 299-310.

(Manuscript received November 11, 1981; revision accepted for publication March 30, 1982.) 